\title{
Editorial: Synthetic Nucleic Acids for Expanding Genetic Codes and Probing Living Cells
}

\author{
Patrick O'Donoghue ${ }^{1,2 *}$, Ilka U. Heinemann ${ }^{1 *}$ and Chenguang Fan ${ }^{3 *}$ \\ ${ }^{1}$ Department of Biochemistry, The University of Western Ontario, London, ON, Canada, ${ }^{2}$ Department of Chemistry, The \\ University of Western Ontario, London, ON, Canada, ${ }^{3}$ Department of Chemistry and Biochemistry, University of Arkansas, \\ Fayetteville, AR, United States
}

Keywords: RNA, synthetic biology, genetic code expansion, fluorescent proteins, RNAi, microRNA, tRNA, non-canonical amino acids

\section{Editorial on the Research Topic}

\section{Synthetic Nucleic Acids for Expanding Genetic Codes and Probing Living Cells}

\section{OPEN ACCESS}

Edited by:

Pasquale Stano,

University of Salento, Italy

Reviewed by:

Thomas E. Gorochowski,

University of Bristol, United Kingdom

${ }^{*}$ Correspondence:

Patrick O'Donoghue

patrick.odonoghue@uwo.ca

Ilka U. Heinemann

ilka.heinemann@uwo.ca

Chenguang Fan

cf021@uark.edu

Specialty section:

This article was submitted to

Synthetic Biology,

a section of the journal

Frontiers in Bioengineering and

Biotechnology

Received: 04 June 2021

Accepted: 07 June 2021

Published: 02 July 2021

Citation:

O'Donoghue P, Heinemann IU and Fan C (2021) Editorial: Synthetic

Nucleic Acids for Expanding Genetic

Codes and Probing Living Cells.

Front. Bioeng. Biotechnol. 9:720534.

doi: 10.3389/fbioe.2021.720534
Synthetic biology is a new and emerging discipline that involves the design and construction of novel biological probes and entities such as enzymes, genetic circuits, or synthetic cells with engineered or expanded biological functions. The field of synthetic biology developed as a result of revolutionary biotechnologies, such as high-throughput DNA synthesis, rapid genome sequencing, as well as directed and continuous evolution approaches to probe cellular functions at the most basic level, including modifying the sequences of nucleic acids and proteins in living cells. Re-writing or re-programming existing biological systems to produce designer proteins and nucleic acid polymers is a major area of focus in synthetic biology.

This special issue of Frontiers in Bioengineering and Biotechnology brings together diverse and cutting-edge applications in synthetic biology. The work highlights the utility, adaptability, and innovative potential of RNAs, including engineered or synthetic RNAs, in revealing biology at the molecular level. Applications of these synthetic nucleic acid technologies span multiple fundamental areas of synthetic biology including genetic code expansion and new fluorescent probes for observations of mistakes in protein synthesis or microRNA activity in live cells.

\section{GENETIC CODE EXPANSION WITH AND WITHOUT CELLS}

Several of the studies in this special issue focused on the area of genetic code expansion. The field involves a range of methods that enable protein synthesis with additional or non-canonical amino acids (ncAA) beyond the standard 20 amino acids building blocks normally used in protein synthesis. Overall, research in this field has led to a staggering array of different chemical functionalities that have applications in site-specific protein labeling with fluorescent or reactive probes, and in protein modifications, such as programmed phosphorylation or acetylation. Indeed, as an introduction to this area, Chung et al. provided an insightful review of the many applications of genetic code expansion, highlighting studies that biochemically characterize specifically labeled or modified proteins.

Pyrrolysyl-tRNA synthetase (PylRS) is a naturally occurring enzyme in certain anaerobic archaea and bacteria that has been used to incorporate $>50$ different ncAAs into proteins, reviewed in Wan et al. (2014). Jiang et al. pushed the enzyme yet further and produced an engineered PylRS mutant with an enhanced activity and the ability to incorporate additional ncAAs, including histidine, and cysteine analogs that have applications in protein engineering and studies of protein function. 


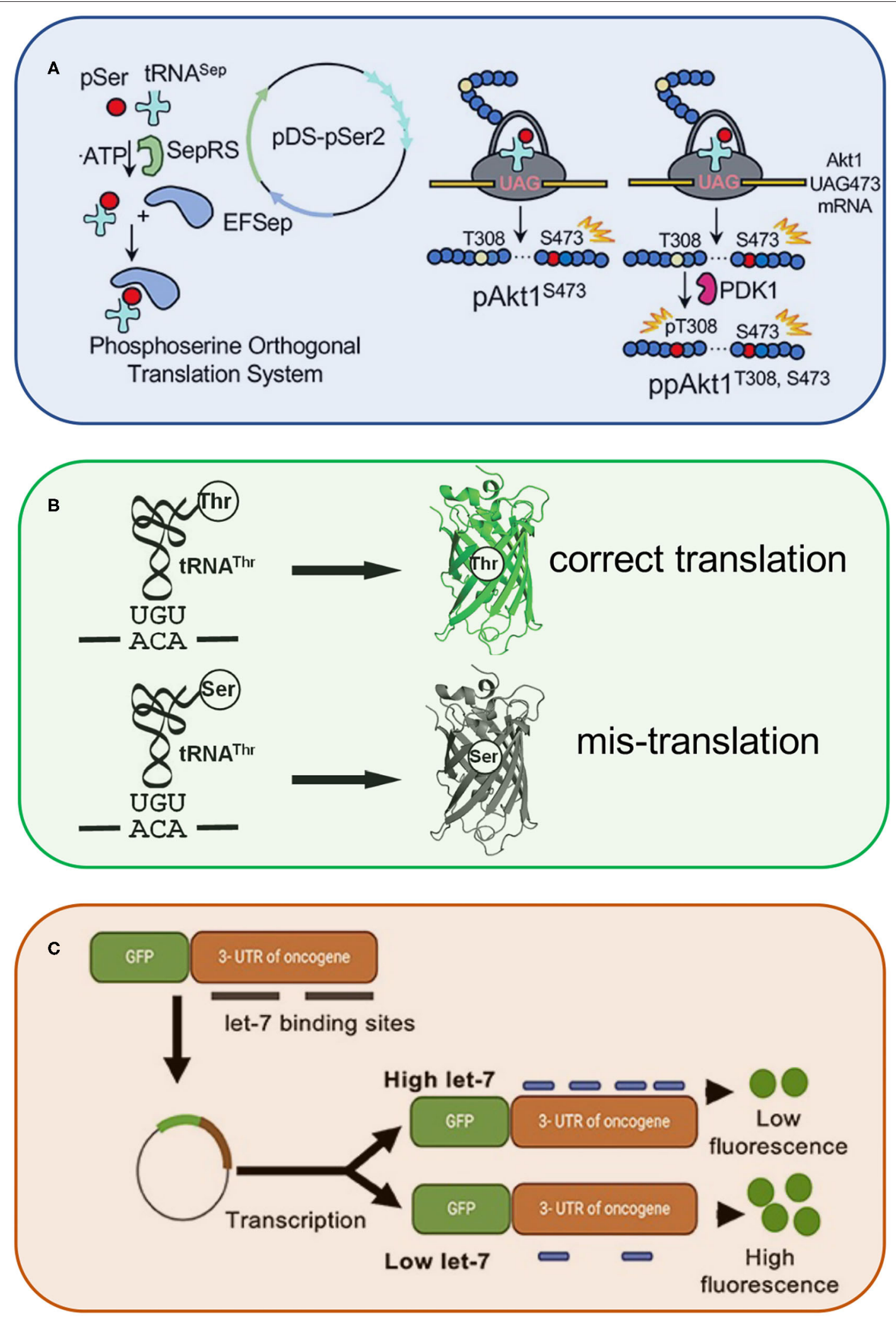

FIGURE 1 | Synthetic and engineered RNAs for genetic code expansion and probing living cells. The schematic highlights a few of the innovative technologies described in this special issue. These include engineered transfer RNAs (tRNA ${ }^{\text {Sep}}$ ) to enable genetic code expansion and applications such as the production of active human kinases (A). In addition, this special issue highlights novel reporters of RNA activity, including green fluorescent protein reporters sensitive to either tRNA-dependent amino acid mis-incorporation (B) or microRNA activity (C). 
Going beyond the barriers to synthetic biology associated with living cells, Cui et al. reviewed advanced cell-free methods for protein synthesis with multiple different ncAAs. Cell-free approaches enable the production of designer proteins with an expanded palette of ncAAs that include diverse chemical functionalities or unusual backbones ( $\beta$-amino acids or $\alpha$ hydroxy acids), including those that might be toxic to cells. Using a cell-free system, Xiao et al. developed a novel approach to generate synthetic proteins with site-specific modifications using a flexizyme. The method employs a flexizyme, which is a nucleic acid enzyme or ribozyme that aminoacylates transfer RNAs (tRNAs) with a desired non-canonical amino acid (ncAA). The authors demonstrated synthesis of histones with site-specific lysine acetylation or incorporation of a non-hydrolysable thioacetyl-lysine analog for studies on the histone code.

Finally, McKenna et al. used genetic code expansion to generate specifically phosphorylated forms of the oncogenic kinase, AKT1 (Figure 1A). The system relies on an engineered transfer RNA (tRNA) (Hohn et al., 2006) that is specifically recognized by phosphoseryltRNA synthetase (SepRS) to reassign UAG codons to phosphoserine. The authors produced active variants of the kinase to validate putative AKT1 substrates (Balasuriya et al., 2020), which represent new targets to inhibit AKT1-dependent oncogenesis.

\section{FLUORESCENT LIVE-CELL REPORTERS FOR MISTRANSLATION AND GENE SILENCING}

Chen et al. engineered a novel fluorescent reporter (Figure 1B) to observe and quantify errors in proteins synthesis in live cells. The technology enabled studies of an editing defective tRNA synthetase that produces both properly aminoacylated as well as misaminoacylated tRNA species leading to mistranslation in bacterial cells.

MicroRNAs are naturally occurring small non-coding RNAs that bind to particular messenger RNAs (mRNAs) for gene silencing. Although the ability of miRNAs to regulate gene expression, including that of oncogenes, is well-known, approaches to measure microRNA activity are often lacking. Siddika and Heinemann reviewed a new and emerging field devoted to engineering protein reporters and new methods to detect and quantify microRNA activity, localization, and quantity (Figure 1C). Next generation RNA sequencing, for example, can uncover unknown microRNAs,

\section{REFERENCES}

Balasuriya, N., Davey, N. E., Johnson, J. L., Liu, H., Biggar, K. K., Cantley, L. C., et al. (2020). Phosphorylation-dependent substrate selectivity of protein kinase B (AKT1). J. Biol. Chem. 295, 8120-8134. doi: 10.1074/jbc.RA119.01 2425 and fluorescent microRNA probes can measure the level of active rather than total microRNA in living cells (Turk et al., 2018).

The ability to precisely manipulate gene expression is important for defining how gene products function and for designing genetic circuits for synthetic biology. Indeed, microRNAs inspired the use of synthetic RNAs to silence gene expression as well. RNA interference (RNAi) is a technology that allows targeted silencing of particular mRNAs with complementary sequences. Because silencing RNAs (siRNAs) are normally produced as a duplex, the opposite or passenger strand, has significant potential to silence off-target genes. Sheng et al. reviewed new approaches to reduce off-target gene silencing using single stranded siRNAs.

In summary, here we highlighted innovate approaches involving engineered or synthetic nucleic acid-based technologies to expand the genetic code with additional amino acids or to observe or manipulate the activity of different kinds of RNAs in living cells. We anticipate that the studies contained in our special issue will inspire new RNA technologies for applications in biomedical research and synthetic biology.

\section{AUTHOR CONTRIBUTIONS}

All authors listed have made a substantial, direct and intellectual contribution to the work, and approved it for publication.

\section{FUNDING}

Work in the authors' laboratories was supported by grants to the Natural Sciences and Engineering Research Council of Canada (04282-2014 to PO'D), Canada Foundation for Innovation (229917 to PO'D), the Ontario Research Fund (229917 to PO'D), Canada Research Chairs (232341 to PO'D), the Canadian Institutes of Health Research (165985 to PO'D), Natural Sciences and Engineering Research Council of Canada (04776-2014 to $\mathrm{IH})$, The Ontario Ministry of Research and Education (ER18-14-193 to IH), The Canadian Rare Diseases: Models \& Mechanisms Network (F19-00472 to IH), and the National Institute of General Medical Sciences (P20GM139768 to CF and R15GM140433 to CF).

\section{ACKNOWLEDGMENTS}

We are especially grateful to each of the authors for their contributions of either an original research article or an inspired review of recent advances to this Research Topic. 
Wan, W., Tharp, J. M., and Liu, W. R. (2014). Pyrrolysyl-tRNA synthetase: an ordinary enzyme but an outstanding genetic code expansion tool. Biochim. Biophys. Acta 1844, 1059-1070. doi: 10.1016/j.bbapap.2014.03.002

Conflict of Interest: The authors declare that the research was conducted in the absence of any commercial or financial relationships that could be construed as a potential conflict of interest.
Copyright (๑ 2021 O'Donoghue, Heinemann and Fan. This is an open-access article distributed under the terms of the Creative Commons Attribution License (CC BY).

The use, distribution or reproduction in other forums is permitted, provided the original author(s) and the copyright owner(s) are credited and that the original publication in this journal is cited, in accordance with accepted academic practice. No use, distribution or reproduction is permitted which does not comply with these terms. 